\title{
The Effect of Hypofractionated Radiotherapy on Tumor Control and Survival in Patients with High-Risk Breast Cancer
}

\author{
Aiat Morsy ${ }^{1}$, Sara H. Hammouda², Samir Shehata ${ }^{1}$ \\ ${ }^{1}$ Clinical Oncology and Nuclear Medicine Department, Faculty of Medicine, Assiut University, Assiut, Egypt \\ ${ }^{2}$ Radiotherapy Department, South Egypt Cancer Institute, Assiut University, Assiut, Egypt \\ Email:*dr_aiat@yahoo.com
}

How to cite this paper: Morsy, A., Hammouda, S.H. and Shehata, S. (2019) The Effect of Hypofractionated Radiotherapy on Tumor Control and Survival in Patients with High-Risk Breast Cancer. Journal of Cancer Therapy, 10, 86-96.

https://doi.org/10.4236/jct.2019.101007

Received: December 30, 2018

Accepted: January 20, 2019

Published: January 23, 2019

Copyright () 2019 by author(s) and Scientific Research Publishing Inc. This work is licensed under the Creative Commons Attribution International License (CC BY 4.0).

http://creativecommons.org/licenses/by/4.0/

\begin{abstract}
BACKGROUND Adjuvant radiotherapy is given following surgery in breast cancer patients. Hypofractonated radiotherapy can significantly reduce the waiting time for radiotherapy, working load on machines, patient visits to radiotherapy departments and medical costs. Material and Methods 244 patients with high-risk breast cancer (stage IIB, stage III and stage IA with any of the following criteria: lymphovascular invasion, hormonal receptor negative, young age) who underwent Breast conservative surgery (BCS) or Modified radical mastectomy (MRM) were enrolled in this study. All patients received adjuvant radiotherapy with different hypofractionation schedules either $3900 \mathrm{cGY} / 13$ fractions or $4240 \mathrm{cGY} / 16$ fractions or $4005 \mathrm{cGY} / 15$ fractions using linear accelerator with $6 \mathrm{MV}$ photon beam. Lateral/Medial tangential and Ipsilateral supraclavicular fields were employed and the ipsilateral axilla was also irradiated if required to the same dose with posteroanterior field. Patients were followed every 3 mons for the first 2 years and every 6 mons thereafter. Outcomes were analyzed in terms of tumor control and survival. Results 244 patients with high-risk breast cancer requiring postoperative radiotherapy to the intact breast or chest wall were treated. The mean age was 48 years (range 28 - 69 years). The 5-year locoregional free survival of all patients was $93.8 \%$ the local relapse reported in 15 patients (6.2\%) 7 patients at site of operated scar \& 8 patients at the regional lymph nodes. The median follow up period was 75 months ranged from 49 to 102 months. Distant metastasis free survival was $92.2 \%$, the distant metastasis reported in 19 patients which represent $(7.8 \%)$ of all patients, median survival is 75 months ranged from 49 to 102 months and overall survival was $88.6 \%$. Conclusion It is concluded that hypofractionated radiotherapy is a simple and effective protocol in patients with high-risk breast cancer regarding tumor control and survival.
\end{abstract}




\section{Keywords}

Breast Cancer, Hypofractionated Radiotherapy, Tumor Control, Survival

\section{Introduction}

Breast cancer is the most common neoplasm in women, accounting for $29 \%$ of all cancers diagnosed annually; overall it is the 2nd leading cause of death following lung cancer. Breast cancer however, is the leading cause of cancer death in women $>65$ years [1].

The choice of therapy depends on tumor characteristics [2]. For example, breast conservation surgery can often be performed, if diagnosed at an early stage. Patients often receive adjuvant radiation therapy following surgery. Studies have reported that breast conserving surgery followed by irradiation yields results similar to those of radical operations such as full mastectomy [3].

In high-risk breast cancer patients, radiotherapy is an important treatment modality. It reduces the risk of local relapse by about $70 \%$ and reduces breast cancer mortality. Normal and malignant tissues vary in their responses to radiotherapy fraction size, termed fractionation sensitivity [4].

The standard conventional radiotherapy delivers a dose fraction of 1.8 - $2 \mathrm{GY}$ per day, for a period of 5 to 7 weeks. In comparison, hypofractionated radiotherapy delivers larger dose radiation fraction over a shorter period of time. Hypofractionated radiotherapy has been shown to provide local tumor control as a logistically more feasible and cost effective treatment protocol [5].

It has been reported that breast cancer has a low a/b ratio ( 4 for breast adenocarcinoma and 3 for normal breast tissue); therefore, a shorter regimen of radiotherapy could theoretically be effective without significantly increasing the adverse effects. Hypofractionated radiotherapy can significantly reduce the waiting time for radiotherapy, working load on machines, patient visits to radiotherapy departments and medical costs [6].

The rational of this study was to evaluate the efficacy of hypofractionated radiotherapy with a dose of 3900 CGY/13 fractions or 4005 CGY/15 fractions or 4240 CGY/16 fractions to the whole breast or chest wall on tumor control and survival in high risk breast cancer in our population.

\section{Patients and Methods}

This study was conducted between January 2010 till December 2014 at the department of radiotherapy, South Egypt cancer institute, Assiut University, Egypt.

The following criteria were used to enroll patients: female gender, patients with age $>18$ and $<75$ years with normal functioning cardiac, renal and pulmonary functions, patients with histopathologically confirmed carcinoma of the unilateral breast without evidence of distant metastasis, patients after surgery either breast conservative surgery or Modified radical mastectomy with high risk 
features (either Stage IIB, Stage III or Stage IIA with any of the following features: lymphovascular invasion, hormonal receptor negative or young age).

Patients with age $>75$ years or who were inoperable or had distant metastasis were excluded from this study.

244 patients from South Egypt cancer institute, all patients underwent complete physical examination, routine blood count, biochemistry, contrast enhanced C.T of the chest and abdomen before starting treatment.

Patients were treated with a linear accelerator using lateral and medial tangential fields to a dose of either $3900 \mathrm{CGY} / 13$ fractions or 4005 CGY/15 fractions or $4240 \mathrm{CGY} / 16$ fractions with $6 \mathrm{MV}$ photon beam, the ipsilateral axilla was included in supraclavicular field and was radiated to the same dose with anteroposterior field using $6 \mathrm{MV}$ photon beam in patients with node positive extracapsular disease in the axilla.

Patient follow up included: patients were examined clinically every week during radiotherapy, every 3 months for the first 2 year and every 6 months thereafter any suspicious lesion was subjected to biopsy and also during follow up patients were subjected to mammography/sonomammograhy/C.T scan/MRI chest wall (in selected cases) to evaluate any local recurrence. Distant metastasis was evaluated by both clinical and imaging examinations.

Data analysis were done with the help of SPSS version 22 software, which included descriptive analysis, Mean was calculated for quantitative variable like age. Locoregional recurrence free survival, distant metastasis free survival and overall survival were calculated.

\section{Results}

Our retrospective study included 244 patients with high-risk breast cancer (Stage IIB, Stage III, Stage IIA with one of the following criteria: lymphovascular invasion, hormonal receptor negative, young age) who underwent either breast conservative surgery or modified radical mastectomy. All of our patients received adjuvant radiotherapy with different hypo-fractionation schedules. Those patients attended to the radiotherapy department of South Egypt cancer institute (SECI), Assuit University, Egypt, between January 2010 till December 2014.

\subsection{Patients' Characteristics}

In our study, the median age of our patients was 48 years with age ranged from 28 to 69 years, $59 \%(\mathrm{n}=144)$ of patients were $<55$ years of age, $81 \%(\mathrm{n}=198)$ had grade 2 disease, and 96\% $(\mathrm{n}=234)$ had infiltrating ductal carcinoma. 63\% ( $=154)$ of patients had T2 disease while 19\% $(\mathrm{n}=46)$ had T3 and $12 \%(\mathrm{n}=29)$ had T4 and only $6 \%(n=15)$ had T1. The histopathological examination of the dissected axillary lymph nodes revealed that $11 \%(\mathrm{n}=27)$ of patients had N0 (negative nodes) and $39 \%(\mathrm{n}=95)$ had $\mathrm{N} 1$ (positive $1-3$ lymph nodes) and $31 \%$ $(\mathrm{n}=76)$ had N2 and 19\% $(\mathrm{n}=46)$ had N3. It was found that all patients $(\mathrm{n}=$ 
244) had negative surgical margins. Regarding the hormonal receptors, our present study showed that $72 \%(n=176)$ of patients had positive hormonal receptors and $28 \%(\mathrm{n}=68)$ had negative hormonal receptors. All patients $(\mathrm{n}=$ 244) received chemotherapy. It was found that $49 \%(n=120)$ of patients had their tumor in the upper outer quadrant while only $7 \%(n=17)$ of patients had their tumor in the central area of the breast. According to the laterality, 53\% ( $\mathrm{n}=$ 129) of patients suffered from left side breast cancer and $47 \%(n=115)$ suffered from right breast cancer. Finally regarding the Her 2/new over-expression, it was found that $19 \%(n=46)$ of the patients had over-expression of the Her $2 /$ new receptors while $81 \%$ of the patients $(n=198)$ did not show Her $2 /$ new over-expression as shown in Table 1.

\subsection{Treatment Characteristics}

In our study, $78 \%(\mathrm{n}=190)$ underwent MRM and 22\% $(\mathrm{n}=54)$ underwent BCS. Regarding chemotherapy 23\% $(\mathrm{n}=56)$ received FAC and 51\% $(\mathrm{n}=125)$ received AC-Taxol and 21\% $(n=51)$ received FEC and 5\% $(n=12)$ received CMF. Regarding radiotherapy $60.6 \%(\mathrm{n}=148)$ received $4240 \mathrm{cGy} / 16$ fraction and $29.4 \%(\mathrm{n}=72)$ received $3900 \mathrm{cGy} / 13$ fraction and $10 \%(\mathrm{n}=24)$ received 4005 cGy/15 fraction. Regarding Hormonal therapy $7.4 \%(n=19)$ received tamoxifen and $49.3 \%(\mathrm{n}=120)$ received aromatase inhibitors and $15.3 \%(\mathrm{n}=37)$ switched from tamoxifen to AI. $28 \%(n=68)$ did not receive hormonal treatment as shown in Table 2.

\subsection{Clinical Outcomes}

\subsubsection{Locoregional Recurrence (LRR)}

In our study, the local relapse reported in 15 patients $(6.2 \%) 7$ patiens at site of operated scar \& 8 patients at the regional lymph nodes. The median follow up period was 75 months ranged from 49 to 102 months, the 5 year locoregional free survival of all patients was $93.8 \%$ as shown in Figure 1.

Univariate analysis for the factors that may affect the disease free survival (DFS) including the age ( $\mathrm{P}$ value 0.432$), \mathrm{T}$ stage $(\mathrm{P}$ value $<0.002$ ), $\mathrm{N}$ stage $(\mathrm{P}$ value 0.001 ), and hormonal receptor status ( $\mathrm{P}$ value 0.001 ), Radiotherapy ( $\mathrm{P}$ value 0.563 ) showed that $\mathrm{T}$ stage, $\mathrm{N}$ stage and Hormonal status had significant effect on the patient LRR free survival as shown in Table 3.

\subsubsection{Distant Metastasis Free Survival}

In our study, the Distant metastasis reported in 19 patients which represent (7.8\%) of all patients, the 5 year distant metastasis free survival was $92.2 \%$ as shown in Figure 2.

Univariate analysis for the factors that may affect the Distant metastasis free survival including the age ( $\mathrm{P}$ value 0.981$)$, T stage $(\mathrm{P}$ value $<0.001), \mathrm{N}$ stage $(\mathrm{P}$ value 0.002 ), and hormonal receptor status ( $\mathrm{P}$ value $<0.001)$, Radiotherapy ( $\mathrm{P}$ value 0.763 ) showed that $\mathrm{T}$ stage, $\mathrm{N}$ stage and Hormonal status had significant effect on the patient DM free survival as shown in Table 4. 


\subsubsection{Overall Survival}

In our study, median survival is 75 months ranged from 49 to 102 months and 5 year overall survival (OS) is $88.6 \%$ as shown in Figure 3.

In Table 5: Univariate analysis for the factors that may affect the overall survival including the age $(\mathrm{P}$ value 0.534$)$, $\mathrm{T}$ stage $(\mathrm{P}$ value $<0.001)$, $\mathrm{N}$ stage ( $\mathrm{P}$ value 0.002 ), and hormonal receptor status ( $\mathrm{P}$ value 0.001 ), Radiotherapy ( $\mathrm{P}$ value 0.984 ) showed that $\mathrm{T}$ stage, $\mathrm{N}$ stage and Hormonal status had significant effect on the patient DM free survival.

Table 1. Patient's characteristics.

\begin{tabular}{|c|c|c|}
\hline Variable & No. & $\%$ \\
\hline \multicolumn{3}{|l|}{ 1) Age at time of diagnosis: } \\
\hline$<55$ years & 144 & $59 \%$ \\
\hline$\geq 55$ years & 100 & $41 \%$ \\
\hline Range & $28-69$ years. & \\
\hline Median & 48 years. & \\
\hline \multicolumn{3}{|l|}{ 2) Laterality: } \\
\hline RT side & 115 & $47 \%$ \\
\hline LT side & 129 & $53 \%$ \\
\hline \multicolumn{3}{|l|}{ 3) Quadrant site } \\
\hline UO (upper outer) & 120 & $49 \%$ \\
\hline UI (upper inner) & 32 & $13 \%$ \\
\hline LO (lower outer) & 49 & $20 \%$ \\
\hline LI (lower inner) & 26 & $11 \%$ \\
\hline $\mathrm{CE}$ (central) & 17 & $7 \%$ \\
\hline \multicolumn{3}{|l|}{ 4) Tumor grade } \\
\hline Grade 1 & 4 & $1.6 \%$ \\
\hline Grade 2 & 198 & $81 \%$ \\
\hline Grade 3 & 42 & $17.4 \%$ \\
\hline \multicolumn{3}{|l|}{ 5) Tumor histopathology } \\
\hline IDC (infiltrating ductal carcinoma) & 234 & $96 \%$ \\
\hline ILC (infiltrating lobular carcinoma) & 10 & $4 \%$ \\
\hline \multicolumn{3}{|l|}{ 6) $\mathrm{T}$ stage: } \\
\hline $\mathrm{T} 1$ & 15 & $6 \%$ \\
\hline $\mathrm{T} 2$ & 154 & $63 \%$ \\
\hline $\mathrm{T} 3$ & 46 & $19 \%$ \\
\hline $\mathrm{T} 4$ & 29 & $12 \%$ \\
\hline \multicolumn{3}{|l|}{ 7) Node stage: } \\
\hline N0 & 27 & $11 \%$ \\
\hline N1 & 95 & $39 \%$ \\
\hline $\mathrm{N} 2$ & 76 & $31 \%$ \\
\hline N3 & 46 & $19 \%$ \\
\hline \multicolumn{3}{|l|}{ 8) Hormonal receptors: } \\
\hline Positive ER and/or PR & 176 & $72 \%$ \\
\hline Negative ER and/or PR & 68 & $28 \%$ \\
\hline \multicolumn{3}{|l|}{ 9) Her 2 new Over-expression: } \\
\hline No & 198 & $81 \%$ \\
\hline Yes & 46 & $19 \%$ \\
\hline
\end{tabular}


Table 2. Treatment characteristics.

\begin{tabular}{ccc}
\hline Variable & No. & $\%$ \\
\hline 1) Surgery: & 190 & $78 \%$ \\
MRM & 54 & $22 \%$ \\
BCS & & \\
2) Chemotherapy: & 56 & $23 \%$ \\
FAC & 51 & $21 \%$ \\
FEC & 125 & $51 \%$ \\
AC-Taxol & 12 & $5 \%$ \\
CMF & & \\
3) Radiotherapy & 148 & $60.6 \%$ \\
4240 cGy/16 fraction & 72 & $29.4 \%$ \\
3900 cGy/13 fraction & 24 & $10 \%$ \\
4005 cGy/15 fraction & & \\
4) Hormonal therapy & & $7.4 \%$ \\
TAM & 19 & $49.3 \%$ \\
AI & 120 & $15.3 \%$ \\
Switched from TAM to AI & 27 & $28 \%$ \\
Not received hormonal therapy & 68 &
\end{tabular}

Table 3. Univariate analysis of the 5 years DFS.

\begin{tabular}{|c|c|c|c|c|}
\hline Variable & & No. of $\mathrm{p}$ & ear DFS\% & $P$ value \\
\hline \multirow{3}{*}{ Age at diagnosis } & $<55$ yrs $(144)$ & 5 & $96.5 \%$ & \multirow{3}{*}{0.432} \\
\hline & & & & \\
\hline & $\geq 55$ yrs $(100)$ & 10 & $90 \%$ & \\
\hline \multirow{5}{*}{$\mathrm{T}$ stage } & $\mathrm{T} 1$ (15) & 1 & $93.5 \%$ & \multirow{5}{*}{0.002} \\
\hline & $\mathrm{T} 2$ (154) & 2 & $98.7 \%$ & \\
\hline & & & & \\
\hline & T3 (46) & 4 & $91.5 \%$ & \\
\hline & T4 (29) & 8 & $73 \%$ & \\
\hline \multirow{5}{*}{ Nodal stage } & No (27) & 0 & $100 \%$ & \multirow{5}{*}{0.001} \\
\hline & N1 (95) & 2 & $98.1 \%$ & \\
\hline & & & & \\
\hline & N2 (76) & 4 & $95.1 \%$ & \\
\hline & N3 (46) & 9 & $80.5 \%$ & \\
\hline \multirow{3}{*}{ Hormonal status } & +ve (176) & 5 & $97.2 \%$ & \multirow{3}{*}{0.001} \\
\hline & & & & \\
\hline & $-\mathrm{ve}(68)$ & 10 & $85.2 \%$ & \\
\hline \multirow{3}{*}{ Radiotherapy } & $\begin{array}{c}4240 / 16 \\
(148)\end{array}$ & 9 & $94.1 \%$ & \multirow{3}{*}{0.563} \\
\hline & $\begin{array}{c}3900 / 13 \\
(72)\end{array}$ & 3 & $96.2 \%$ & \\
\hline & $\begin{array}{c}4005 / 15 \\
(24)\end{array}$ & 3 & $87.5 \%$ & \\
\hline
\end{tabular}


Table 4. Univariate analysis of the 5 years Distant metastasis free survival.

\begin{tabular}{|c|c|c|c|c|}
\hline Variable & & No. of patient & $\begin{array}{c}5 \text { year DM } \\
\text { FS\% }\end{array}$ & $P$ value \\
\hline \multirow{2}{*}{ Age at diagnosis } & $<55$ yrs $(144)$ & 10 & $93.1 \%$ & \multirow{2}{*}{0.981} \\
\hline & $\geq 55$ yrs $(100)$ & 9 & $91 \%$ & \\
\hline \multirow{4}{*}{ T stage } & T1 (15) & 0 & & \multirow{4}{*}{$<0.001$} \\
\hline & T2 (154) & 5 & $96.8 \%$ & \\
\hline & T3 (46) & 6 & $86.95 \%$ & \\
\hline & T4 (29) & 8 & $72.5 \%$ & \\
\hline \multirow{4}{*}{ Nodal stage } & No (27) & 1 & $96.3 \%$ & \multirow{4}{*}{0.002} \\
\hline & N1 (95) & 3 & $96.9 \%$ & \\
\hline & N2 (76) & 4 & $94.8 \%$ & \\
\hline & N3 (46) & 11 & $82.8 \%$ & \\
\hline \multirow{2}{*}{ Hormonal status } & +ve (176) & 6 & $96.6 \%$ & \multirow{2}{*}{$<0.001$} \\
\hline & -ve (68) & 13 & $81.1 \%$ & \\
\hline \multirow{3}{*}{ Radiotherapy } & $\begin{array}{c}4240 / 16 \\
(148)\end{array}$ & 10 & $93.2 \%$ & \multirow{3}{*}{0.763} \\
\hline & $\begin{array}{c}3900 / 13 \\
(72)\end{array}$ & 5 & $93.4 \%$ & \\
\hline & $\begin{array}{c}4005 / 15 \\
(24)\end{array}$ & 4 & $83.3 \%$ & \\
\hline
\end{tabular}

Table 5. Univariate analysis of the 5 years overall survival.

\begin{tabular}{|c|c|c|c|c|}
\hline Variable & & No. of patient & 5 year OS\% & $P$ value \\
\hline \multirow{2}{*}{ Age at diagnosis } & $<55$ yrs $(144)$ & 13 & $90 . .9 \%$ & \multirow{2}{*}{0.534} \\
\hline & $\geq 55$ yrs $(100)$ & 15 & $85 \%$ & \\
\hline \multirow{4}{*}{$\mathrm{T}$ stage } & T1 (15) & 2 & $86.7 \%$ & \multirow{4}{*}{$<0.001$} \\
\hline & T2 (154) & 5 & $96.7 \%$ & \\
\hline & T3 (46) & 7 & $84.7 \%$ & \\
\hline & T4 (29) & 14 & $52 \%$ & \\
\hline \multirow{4}{*}{ Nodal stage } & N0 (27) & 1 & $96.3 \%$ & \multirow{4}{*}{0.002} \\
\hline & N1 (95) & 6 & $93.7 \%$ & \\
\hline & N2 (76) & 8 & $89.5 \%$ & \\
\hline & N3 (46) & 13 & $71 / 7 \%$ & \\
\hline \multirow[b]{2}{*}{ Hormonal status } & +ve (176) & 8 & $95.4 \%$ & \multirow[b]{2}{*}{0.001} \\
\hline & $-\mathrm{ve}(68)$ & 20 & $70.5 \%$ & \\
\hline \multirow{3}{*}{ Radiotherapy } & $\begin{array}{c}4240 / 16 \\
(148)\end{array}$ & 11 & $92.5 \%$ & \multirow{3}{*}{0.984} \\
\hline & $\begin{array}{c}3900 / 13 \\
(72)\end{array}$ & 9 & $87.5 \%$ & \\
\hline & $\begin{array}{c}4005 / 15 \\
(24)\end{array}$ & 8 & $67 \%$ & \\
\hline
\end{tabular}




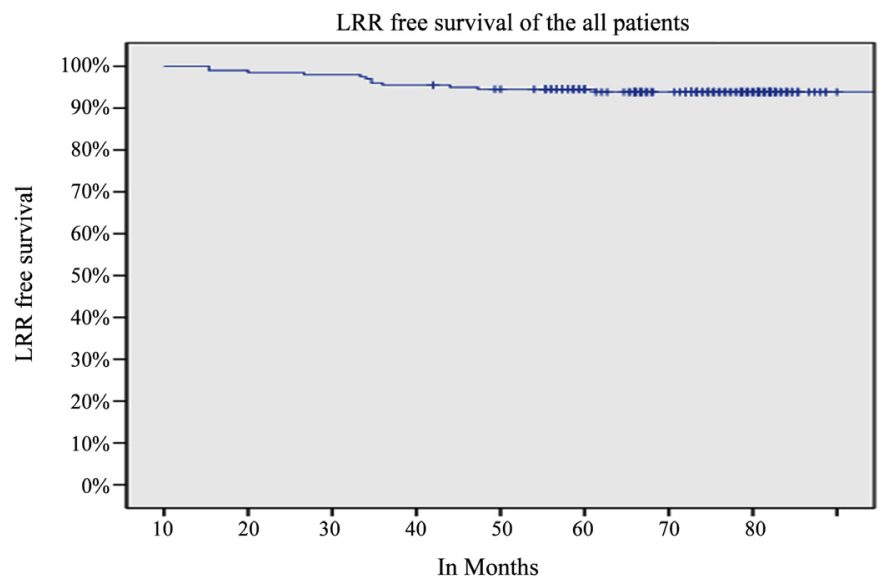

Figure 1. Locoregional free survival.

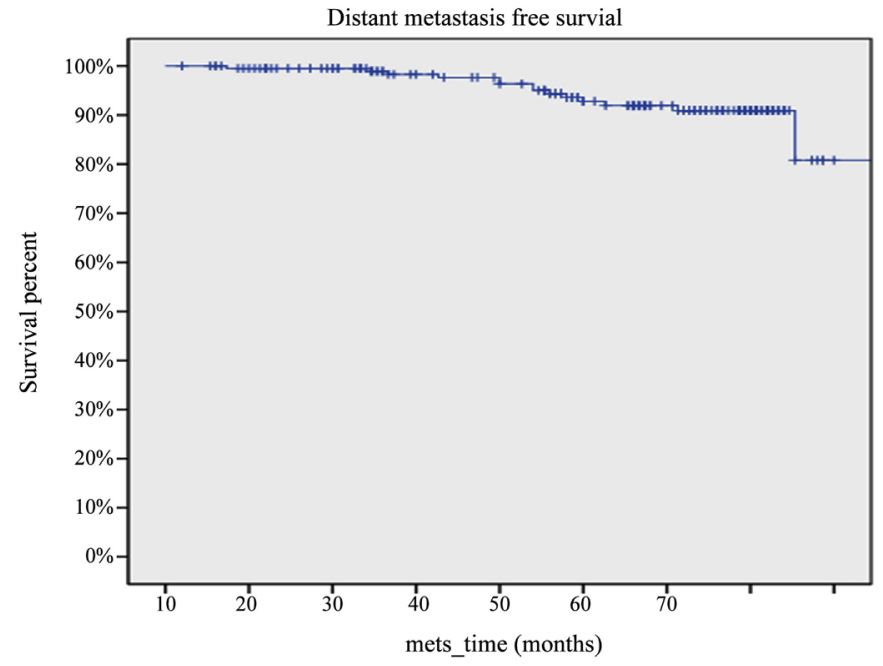

Figure 2. Distant metastasis free survial.

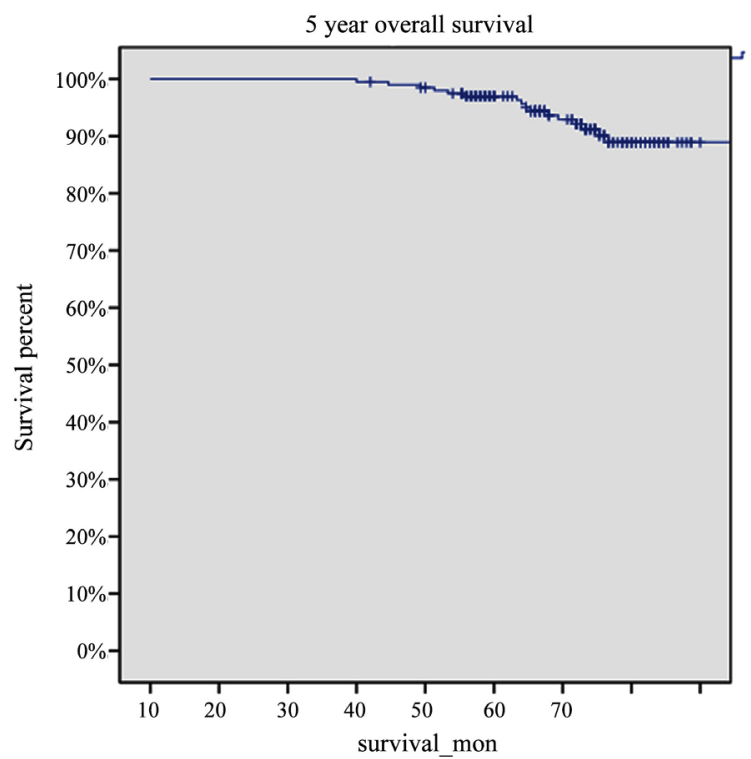

Figure 3. 5 year overall survival. 


\section{Discussion}

Radiotherapy is an essential part of treatment in breast cancer after surgery and chemotherapy. It has been confirmed that adjuvant radiotherapy remarkably improves locoregional control in high-risk patients. After surgery and chemotherapy patients in need for radiotherapy can either be treated with radiotherapy using conventional or altered fractionation schedule. Conventional fractionation delivers radiation over a period of 5 - 7 weeks. This results in an increase in departmental workload and leads to poor compliance on part of patient. So there was an increased need to use a schedule of delivering RT in a shorter time period with equal efficacy and acceptable toxicity. The main concern was toxicity which will result from delivering larger dose per fractionation. However, clinical research and advances in radiobiological principles have provided a number of shorter RT protocols i.e. HFRT. In comparison with conventional RT, hypofractionated radiotherapy has achieved equivalent efficacy and acceptable toxicity [7].

Therefore, we used HFRT protocol with different doses either $3900 \mathrm{CGY} / 13$ fractions or 4005 CGY/15 fractions or 4240 CGY/16 fractions in our institute and observed the efficacy in terms of tumor control and survival.

To compare hypofractionated radiotherapy and conventional radiotherapy several clinical trials have been done. Whelan et al. [8] studied a clinical cancer group in Ontario and it was found that hypofractionated radiotherapy was of benefit in early stage breast cancer. They found that hypofractionated radiotherapy (42.5 Gy in 16 fractions over 22 days) was similar to conventional radiotherapy (50 Gy in 25 fractions over 35 days) regarding early skin toxicity, local recurrence within 10 years, or cosmetic outcome.

The British Columbia Cancer Centerinvestigated local control [9]. In 2012, 1335 grade 3 breast cancer patients (T1-T2, N0, M0) were studied, 252 patients underwent conventional fractionation of $45-50 \mathrm{~Gy}$ in 25 fractions and $1083 \mathrm{pa}-$ tients received a hypofractionated schedule of 42.5 - $44 \mathrm{~Gy}$ in 16 fractions. The 10 -year local relapse cumulative incidence was $6.9 \%$ in the hypofractionated group and $6.2 \%$ in the conventionally fractionated group $(\mathrm{p}=0.99)$. So this study showed that there were no significant differences between the hypofractionated schedule and conventional fractionation in terms of local recurrence, even for histologic grade 3 breast tumors.

Also, two large trials reported similar findings; START A and START B [10]. These two studies compared between hypofractionated radiotherapy and conventionally fractionated radiotherapy in terms of long term local relapse rates. Similar results were achieved with both hypofractionated radiotherapy and conventional radiotherapy.

Another short RT schedule, 40 Gy in 15 fractions, has been used at Christie Hospital in Manchester, UK. They reported results of 2159 treated patients that are comparable to those reported from other centers i.e. LRR up to $11 \%$ [7]. After the encouraging results of the START trials this protocol is now becoming 
the standard in the UK. Other non-randomized studies have reported similar results. Fujii et al. [5] reported equivalent results in terms of LRC up to $90 \%$ with a short fractionation schedule of 42.5 - $47.8 \mathrm{~Gy} / 16$ - 20 fractions. Whelan et al. [8] [11] yielded LRC up to $96.8 \%$ with HFRT protocol. LRC in our study was 93.8\%.

Our study showed that patients prefer hypofractionated radiotherapy because the cost of treatment is significantly lower and it has the advantages of acceptable cosmetic outcomes, acceptable local control, good survival rates, saving in cost and resources which indicate that hypofractionated radiotherapy is an acceptable alternative treatment to conventional radiotherapy.

The potential limitations of our study were there was only one arm of patients, number of patients who underwent modified radical mastectomy were more than patients who underwent breast conservative surgery and a longer period of follow up would be more beneficial. We recommend similar studies using two arms of patients, with more early breast cancer patients undergoing breast conservative surgery and a longer follow up period in the future.

\section{Conclusion}

It is concluded from our study that hypofractionated radiotherapy is a simple, effective and safe treatment protocol for high-risk breast cancer with benefit for survival and tumor control. After the reported data from international studies hypofractionated radiotherapy should be used as standard as its resource saving and logistically convenient.

\section{Conflicts of Interest}

The authors declare no conflicts of interest regarding the publication of this paper.

\section{References}

[1] Chmielowski, B. and Territo, M.C. (2017) Manual of Clinical Oncology. Wolters Kluwer.

[2] Bride, M.B., Neal, L., Dilaveri, C.A., Sandhu, N.P., et al. (2013) Factors Associated with Surgical Decision Making in Women with Early-Stage Breast Cancer: A Literature Review. Journal of Women's Health, 22, 236-242. https://doi.org/10.1089/jwh.2012.3969

[3] Keating, N.L., Landrum, M.B., Brooks, J.M., et al. (2011) Outcomes Following Local Therapy for Early-Stage Breast Cancer in Non-Trial Populations. Breast Cancer Research and Treatment, 125, 803-813. https://doi.org/10.1007/s10549-010-0865-4

[4] Bentzen, S.M., Agrawal, R.K., Aird, E.G., Barrett, J.M., Barrett-Lee, P.J., Bliss, J.M., Brown, J., Dewar, J.A., Dobbs, H.J., Haviland, J.S. and Hoskin, P.J. (2008) The UK Standardisation of Breast Radiotherapy (START) Trial B of Radiotherapy Hypofractionation for Treatment of Early Breast Cancer: A Randomised Trial. Lancet, 371, 1098-1107. https://doi.org/10.1016/S0140-6736(08)60348-7

[5] Fujii, O., Hiratsuka, J., Nagase, N., Tokiya, R., Yoden, E., Sonoo, H., et al. (2008) Whole Breast Radiotherapy with Shorter Fractionation Schedules for Breast Conserving Surgery: Short-Term Morbidity and Preliminary Outcomes. Breast Cancer, 
15, 86-92. https://doi.org/10.1007/s12282-007-0010-3

[6] Hashemi, F.A., et al. (2016) Comparison of Conventional and Hypofractionated Radiotherapy in Breast Cancer Patients in Terms of 5-Year Survival, Locoregional Recurrence, Late Skin Complications and Cosmetic Results. Asian Pacific Journal of Cancer Prevention, 17, 4819.

[7] Riaz, O., Mahmood, A., Alvi, Z.A., Gul, S., Rasul, S. and Haider, N. (2017) Efficacy of Hypofractionated Radiotherapy in Loco-Regional Tumor Control in Breast. Pakistan Armed Forces Medical Journal, 1, 72.

[8] Whelan, T.J., Pignol, J.P., Levine, M.N., et al. (2010) Long-Term Results of Hypofractionated Radiation Therapy for Breast Cancer. New England Journal of Medicine, 362, 513-520. https://doi.org/10.1056/NEJMoa0906260

[9] Herbert, C., Nichol, A., Olivotto, I., et al. (2012) The Impact of Hypofractionated Whole Breast Radiotherapy on Local Relapse in Patients with Grade 3 Early Breast Cancer: A Population-Based Cohort Study. International Journal of Radiation Oncology Biology Physics, 82, 2086-2092. https://doi.org/10.1016/j.ijrobp.2011.01.055

[10] Haviland, J.S., Owen, J.R., Dewar, J.A., et al. (2013) The UK Standardisation of Breast Radiotherapy (START) Trials of Radiotherapy Hypofractionation for Treatment of Early Breast Cancer: 10-Year Follow-Up Results of Two Randomised Controlled Trials. Lancet Oncology, 14, 1086-1094.

https://doi.org/10.1016/S1470-2045(13)70386-3

[11] Whelan, T., MacKenzie, R., Julian, J., Levine, M., Shelley, W., Grimard, L., et al. (2002) Randomized Trial of Breast Irradiation Schedules after Lumpectomy for Women with Lymph Node-Negative Breast Cancer. Journal of the National Cancer Institute, 94, 1143-1150. https://doi.org/10.1093/jnci/94.15.1143 